\title{
Structure and temporal change of the credit network between banks and large firms in Japan
}

\author{
Yoshi Fujiwara ${ }^{1}$, Hideaki Aoyama ${ }^{2}$, Yuichi Ikeda ${ }^{3}$, \\ Hiroshi Iyetomi ${ }^{4}$, Wataru Souma ${ }^{1}$ \\ ${ }^{1}$ ATR/NiCT CIS Applied Network Science Laboratory, Kyoto 619-0288, Japan \\ 2 Department of Physics, Kyoto University, Kyoto 606-8501, Japan \\ ${ }^{3}$ Hitachi Ltd, Hitachi Research Laboratory, Ibaraki 319-1221, Japan \\ ${ }^{4}$ Department of Physics, Niigata University, Ikarashi, Niigata 950-2181, Japan
}

\begin{abstract}
We present a new approach to understanding credit relationships between commercial banks and quoted firms, and with this approach examine the temporal change in the structure of the Japanese credit network from 1980 to 2005. At each year, the credit network is regarded as a weighted bipartite graph where edges correspond to the relationships and weights refer to the amounts of loans. Reduction in the supply of credit affects firms as debtor, and failure of a firm influences banks as creditor. To quantify the dependency and influence between banks and firms, we propose a set of scores of banks and firms, which can be calculated by solving an eigenvalue problem determined by the weight of the credit network. We found that a few largest eigenvalues and corresponding eigenvectors are significant by using a null hypothesis of random bipartite graphs, and that the scores can quantitatively describe the stability or fragility of the credit network during the 25 years.
\end{abstract}

JEL: E51, E52, G21

Keywords: Banking, Credit topology, Bipartite network, Systemic risk

Correspondence: Yoshi Fujiwara, ATR CIS Applied Network Science Lab, Kyoto 619-0288, Japan. Email: yoshi.fujiwara@gmail.com

We would like to thank M. Gallegati and G. De Masi for discussion during a preliminary stage of this work. Y. F. thank them for the collaboration (De Masi et al., 2008). We acknowledge the Nikkei Media Marketing, Inc. for technical assistance. 


\section{Introduction}

The credit-debt relation between banks and firms is one of the most important relationships among economic agents. Credit is a source of profit for a bank, and it is fuel for growth of a firm. The flip side of the relation is, however, the path where failures take place and their propagation occurs often at a nation-wide scale, and sometimes to a world-wide extent, as we experience today.

It is well known that the Japanese banking system suffered a considerable deterioration in its financial condition during the 1990s. Financial institutions in private-sector had accumulated loan losses, more than 80 trillion yen (nearly $15 \%$ of GDP), which reduced the bank capitalization, and led to the failure of three major and other small banks. Even though two major banks were nationalized in 1997, and other political decisions were made in order to maintain the stability of financial system, most banks, major and minor, decreased the supply of credit immediately; even by reducing existing loans to firms. A lot of firms, especially small and medium-sized firms, eventually suffered loss of funding. See Brewer et al. (2003).

Financial systems are, at an aggregate level, subject to the tails of distributions for economic variables. This perspective has been recognized increasingly in economics; personal income, firm-size, number of relationships among firms and banks (ownership, suppliercustomer, etc.), and so on. It has been recognized that distributions and fluctuations are the keys for understanding many phenomena in macro-economy (see Aoki and Yoshikawa (2007) and Delli Gatti et al. (2008)).

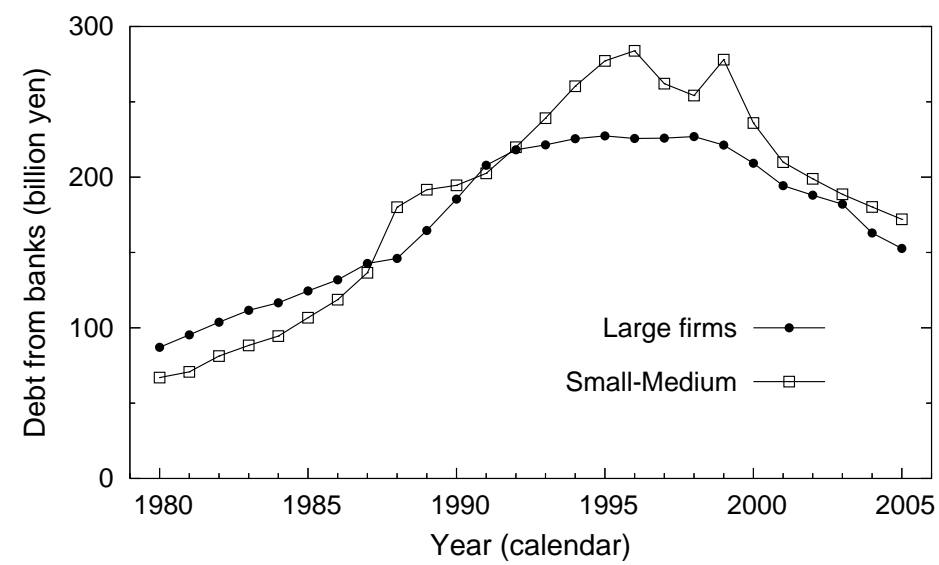

Figure 1: Historical data of the total amount of debt from banks during the calendar years, 1980 to 2005 . For large firms (filled circles) and for small and medium firms (squares).

Fig. 1 shows the historical data of the total amount of debt from banks for large firms and for small and medium firms ${ }^{1}$. For the year 2005, $1.25 \%(33,833)$ of domestic firms are the large firms according to the classification, while the rest $98.75 \%$ are the small-medium firms ${ }^{2}$. Yet the total loans for the large firms amount to be 160 billion yen, which is nearly

\footnotetext{
${ }^{1}$ Source: 2008 white papers on small and medium enterprises in Japan, Small and Medium Enterprise Agency. Here large firms are the companies capitalized at 100 million yen or more, and small-medium firms are the others. Calendar years are used here and throughout this paper.

${ }^{2}$ Source: statistics of corporations by industry, annual report, 1980 to 2005, Ministry of Finance.
} 
equal to those for the small-medium firms as shown in the figure. Thus, only a small fraction of firms account for half of all loans. Conversely, as we shall show in this paper, a large part of loans is provided by a few large banks - the tail of another distribution.

Suppose a large firm is heavily indebted with banks. Then a failure of the firm, or a default, may cause a considerable effect on the balance sheets of the banks. If the banks reduce their supply of credit, then the total supply of loans will be decreased resulting in the adverse shocks to other firms. Therefore, the study of structure of credit relationships or credit network between banks and firms, and its temporal change would give us an insight to understand the financial stability or fragility. This is precisely the purpose of this paper.

There are several related works in the literature. For example, Ogawa et al. (2007) carried out an analysis of dependency of the number of long-term credit relationships on characteristics of firms. Uchida et al. (2008) studied the relation between bank-size and credit links. Kano et al. (2006) investigated the credit of small and medium-sized firms. Studies such as Ogawa et al. (2007) focus on multiple lending relationships. Recently, complex network analysis (see Caldarelli (2007) and references therein) has been applied to financial systems (e.g., Inaoka et al. (2004), Imakubo and Soejima (2008), Iori et al. (2007) for inter-bank relationships, De Masi and Gallegati (2007), De Masi et al. (2008) for bank-firm relationships). In this paper, we shall study on the credit network between banks and large firms by regarding the network as a weighted bipartite graph, develop quantification of fragility of banks, and apply it to credit networks in Japan for the past 25 years.

In Section II, we describe our credit network dataset. In Section A, we consider a credit network as a weighted bipartite graph, and show several statistical properties of heavy-tailed distributions. Then, in Section B, we propose a set of scores for banks and firms which measure potential influences that one agent exerts on the other. It is shown that the scores can be calculated by solving an eigenvalue problem. In Section C, we apply this method to our dataset from the year 1980 to 2005. The results are discussed in Section IV. Appendix A: is for proving mathematical properties for the eigenvalue problem which appeared in Section B.

\section{Dataset}

Our dataset is based on a survey of firms quoted in the Japanese stock-exchange markets (Tokyo, Osaka, Nagoya, Fukuoka and Sapporo, in the order of market size). The data were compiled from the firms' financial statements and survey by Nikkei Media Marketing, Inc. in Tokyo, and are commercially available. They include the information about each firm's borrowing obtained from financial institutions such as the amounts of borrowing and their classification into short-term and long-term borrowings. We examined the period from the years 1980 to 2005, for which incomplete data are few, and study the time development of credit relationships by using the total of long and short-term credit.

For financial institutions, we select commercial banks as a set of leading suppliers of credit. The set comprises long-term, city, regional (primary and secondary), trust banks, insurance companies and other institutions including credit associations. During the examined period, more than 200 commercial banks existed, which are summarized in Table 1. We remark that failed banks are included until the year of failure, and that merger and acquisition of banks are processed consistently. For quoted firms, we choose only surviving firms that are quoted in the stock markets mentioned above ${ }^{3}$.

\footnotetext{
${ }^{3}$ Based on the lists of surviving firms and quoted firms in September and December 2007 respectively. Firms registered on over-the-counter (OTC) market and/or on JASDAQ (the present OTC market) are
} 


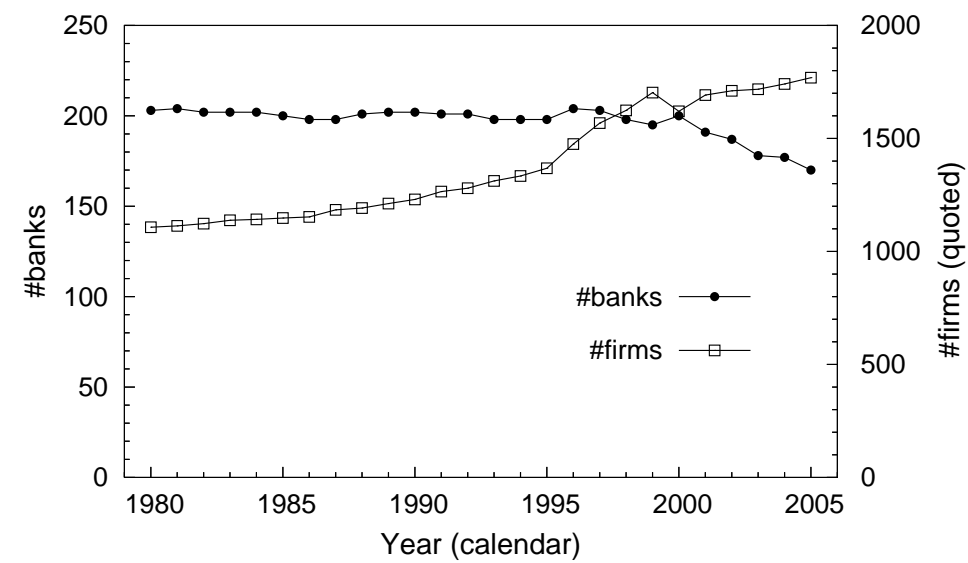

Figure 2: The number of commercial banks and quoted firms.

Table 1: Classification of commercial banks. \# denotes the net number of institutions in each corresponding category during the years, 1980 to 2005. The leftmost column, a to $j$, is defined as a short-hand notation.

\begin{tabular}{|c|l|r|}
\hline id & Classification & $\#$ \\
\hline \hline$a$ & Long-term credit banks & 3 \\
$b$ & City banks & 16 \\
$c$ & Regional banks & 64 \\
$d$ & Secondary regional banks & 71 \\
$e$ & Trust banks & 20 \\
$f$ & Life insurance companies & 23 \\
$g$ & Non-life insurance companies & 23 \\
$h$ & Credit associations (Shinkin banks) & 4 \\
$i$ & Agricultural financial institutions & 4 \\
$j$ & Shoko Chukin bank & 1 \\
\hline & Total & 229 \\
\hline
\end{tabular}

The number of banks and firms in each year is summarized in Fig. 2. The classification of banks and industrial sectors of firms are shown in Table 1 and Table 2 respectively.

The number of banks and firms in each year is summarized in Fig. 2. The classification of banks and industrial sectors of firms are shown in Table 1 and Table 2 respectively.

excluded. The dataset includes the OTC and JASDAQ data since 1996, so we exclude them also by checking the listing date of the firms added in the dataset. 
Table 2: Sectors of quoted firms in the dataset. \# denotes the net number of firms in each sector during the years, 1980 to 2005. The total number of the firms amounts to 2,330 .

\begin{tabular}{|l|r||l|r|}
\hline manufacturing & $\#$ & non-manufacturing & $\#$ \\
\hline \hline Foods & 105 & Marine products & 5 \\
Textile products & 60 & Mining & 7 \\
Pulp \& paper & 18 & Construction & 148 \\
Chemicals & 156 & Wholesale trade & 233 \\
Drugs \& medicines & 33 & Retail trade & 153 \\
Petroleum \& coal & 11 & Securities & 18 \\
Rubber products & 20 & Credit \& leasing & 75 \\
Ceramic, etc. & 49 & Real estate & 75 \\
Iron \& steel & 49 & Railway transport. & 27 \\
Non-ferrous metals & 106 & Road transport. & 28 \\
General machinery & 182 & Water transport. & 15 \\
Electronics & 203 & Air transport. & 4 \\
Shipbuilding & 6 & Warehousing & 38 \\
Motor vehicles & 65 & Information Tech. & 20 \\
Transportation equip. & 11 & Utilities (electric) & 11 \\
Precision instruments & 40 & Utilities (gas) & 13 \\
Other manufacturing & 82 & Services & 264 \\
\hline
\end{tabular}

\section{Analysis of Credit Network}

\section{A. Credit Network as a Weighted Bipartite Graph}

Each yearly statement, or snapshot, of the credit network in our dataset can be regarded as a bipartite graph. Nodes are either banks or firms ${ }^{4}$. Banks and firms are denoted by Greek letters $\mu(\mu=1, \ldots, n)$ and Latin letters $i(i=1, \ldots, m)$ respectively. $n$ is the number of banks, and $m$ is that of firms. An edge between a bank $\mu$ and a firm $i$ is defined to be present if there is a credit relationship between them. In addition, a positive weight $w_{\mu i}$ is associated with the edge, which is defined to be the amount of the credit. We can depict the network as shown in Fig. 3.

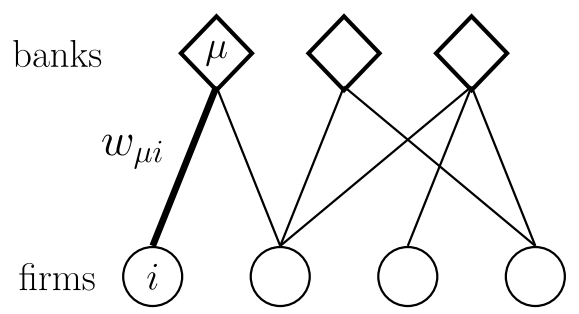

Figure 3: Credit network as a bipartite graph. An edge connecting between bank $\mu$ and firm $i$ is associated with an amount of credit $w_{\mu i}$ as a weight.

\footnotetext{
${ }^{4}$ Note that banks are not included in the side of firms, even if they are borrowing from other banks. Because our dataset includes banks' borrowing only partially, the interbank credit is not considered here, though it is no less important than the bank-firm credit studied here.
} 
$w_{\mu i}$ is the amount of lending by bank $\mu$ to firm $i$, which precisely equals to the amount of borrowing by firm $i$ from bank $\mu$. The total amount of lending by bank $\mu$ is

$$
w_{\mu}:=\sum_{i} w_{\mu i}
$$

and the total amount of borrowing by firm $i$ is

$$
w_{i}:=\sum_{\mu} w_{\mu i}
$$

We note that a same value $w_{\mu i}$ has different meanings as a weight to the bank $\mu$ and to the firm $i$. For example, even if $90 \%$ of the total lending of the bank $\mu$ goes to the firm $i$, it may be the case that $i$ depends on $\mu$ by only $20 \%$ for all the loans from banks. It would be natural to define an $(n \times m)$ matrix $\mathbf{A}$ whose component is given by

$$
A_{\mu i}:=\frac{w_{\mu i}}{w_{\mu}} .
$$

$A_{\mu i}$ represents the relative amount of lending by bank $\mu$ to firm $i$. We have

$$
\sum_{i} A_{\mu i}=1 \quad \text { for all } \mu
$$

Similarly, we define an $(m \times n)$ matrix $\mathbf{B}$ by

$$
B_{i \mu}:=\frac{w_{\mu i}}{w_{i}} .
$$

$B_{i \mu}$ represents the relative amount of borrowing by firm $i$ from bank $\mu$. We have

$$
\sum_{\mu} B_{i \mu}=1 \text { for all } i
$$

The degree $k_{\mu}$ of bank $\mu$ is the number of edges emanating from it to firms, and the degree $k_{i}$ of firm $i$ is the number of edges to banks. When the weights $w_{\mu i}$ are all equal to 1 , it is obvious that $k_{\mu}=w_{\mu}$ and $k_{i}=w_{i}$.

The distributions for $w_{\mu}, w_{i}, k_{\mu}, k_{i}$ have long-tails. They are shown, for the data of credit relationships in the year 2005, in Fig. 4 (a) to (d). The long-tails for the banks' amount of credit and number of firms for lending, in Fig. 4 (a) and (c) for $w_{\mu}$ and $k_{\mu}$ respectively, are comprised of city banks, long-term credit banks, several of trust banks and insurance companies (see the classification in Table 1). Similar long-tails are observed for firms, as shown in Fig. 4 (b) and (d) for $w_{i}$ and $k_{i}$.

There is a significant correlation between $w_{\mu}$ and $k_{\mu}$ in a natural way, and also for $w_{i}$ and $k_{i}$, as shown in Fig. 4 (e) and (f) respectively. We calculated rank correlation in terms of Kendall's $\tau$, which gave significant values of $\tau=0.825(16.0 \sigma)$ and $\tau=0.450(28.3 \sigma)$ respectively, where $\sigma$ is the value under the null hypothesis of statistical independence. In particular, from the Fig. 4 (e), we can observe an empirical relation of $k_{\mu} \propto w_{\mu}^{a}$, where $a \approx$ $0.69 \pm 0.03$ (least-square fit; error $95 \%$ level). This implies the relation of $w_{\mu} / k_{\mu} \propto k_{\mu}^{0.44 \pm 0.07}$ meaning that the average loan is larger for the larger degree $k_{\mu}$, or roughly speaking, for the larger banks. This observation is consistent with known empirical facts (see Uchida et al. (2008) on similar relation for borrowing by small and medium-sized enterprises).

We refer the reader to De Masi et al. (2008) for extensive study on statistical properties of credit topology and weights. 

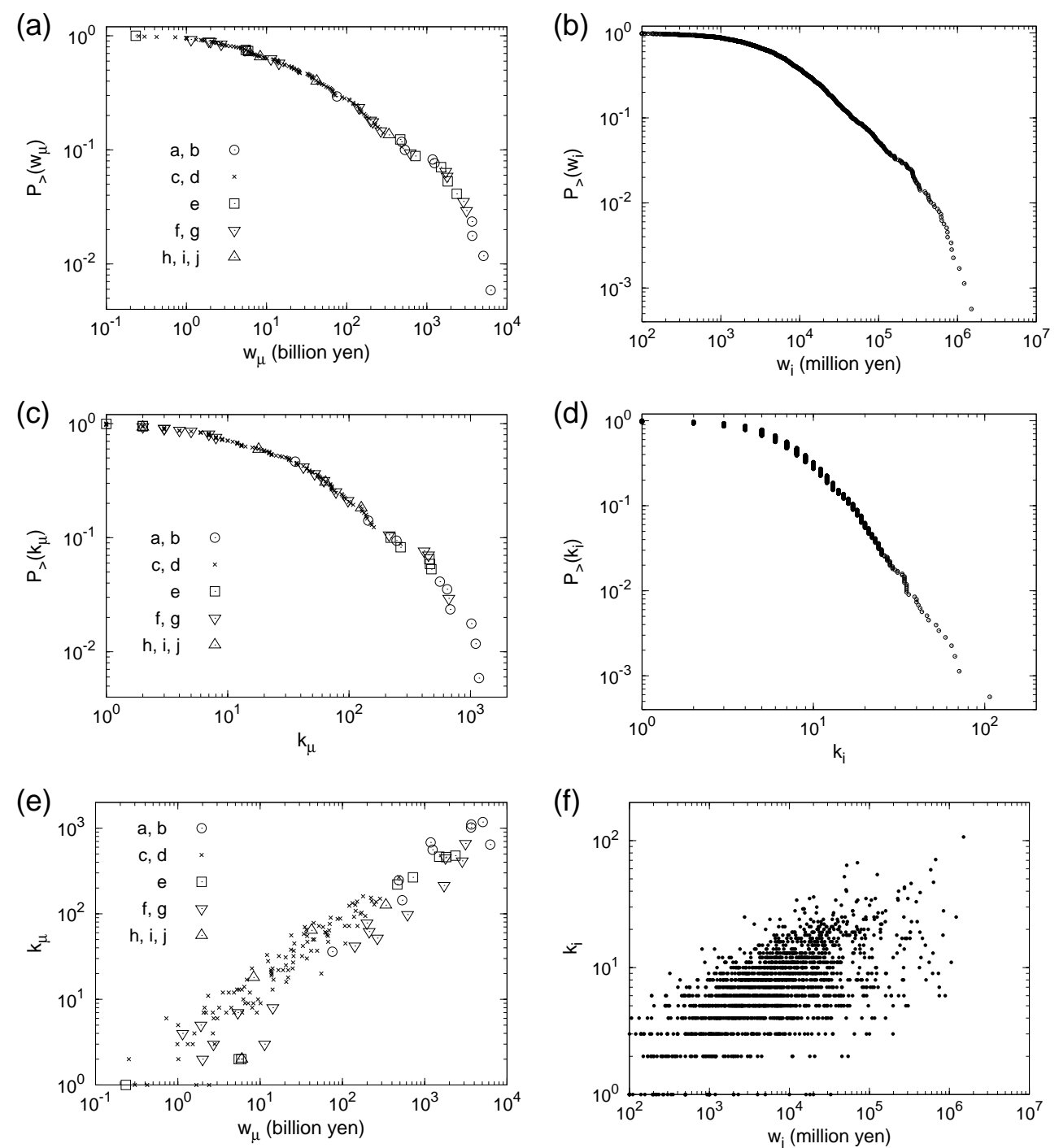

Figure 4: (a) Cumulative distribution $P_{>}\left(w_{\mu}\right)$ for banks' lending $w_{\mu}$. (b) $P_{>}\left(w_{i}\right)$ for firms' borrowing. (c) $P_{>}\left(k_{\mu}\right)$ for the number of banks' lending relationships. (d) $P_{>}\left(k_{i}\right)$ for the number of firms' borrowing relationships. (e) Scatter plot for banks' $w_{\mu}$ and $k_{\mu}$. (f) Scatter plot for firms' $w_{i}$ and $k_{i}$. All the plots are for the data in the year 2005. In the plots (a),(c) and (e) for banks, the points are drawn according to the classification given in Table 1. Rank correlations (Kendall's $\tau$ ) for (e) and (f) are $\tau=0.825(16.0 \sigma)$ and $\tau=0.450(28.3 \sigma)$ respectively ( $\sigma$ calculated under the null hypothesis of statistical independence). 


\section{B. Fragility Scores of Banks}

Bank and firm establish a credit relationship for obvious reasons. Bank supplies credit in anticipation of interest margin, and firm uses credit as an important source of financing in anticipation of growth in its business. An edge of credit, therefore, represents dependency of one agent on the other in two ways.

$A_{\mu i}$ quantifies the dependency of bank $\mu$ on firm $i$ as a source of profit. Also $B_{i \mu}$ is the dependency of firm $i$ on bank $\mu$ as a source of financing from financial institutions. The flip side of dependency is a potential influence which one agent exerts on the other, as we argue below.

Suppose that one can quantify a change in the level of bank $\mu$ 's financial deterioration by a variable or score, $x_{\mu}$, which is to be defined in a consistent way by the following argument. Bank $\mu$ with increasing $x_{\mu}$ will behave in various ways; it may shrink the amount of its supplied credit, increase interest-rate, shorten the due time of payment by firms, and so forth. In any case, it would influence firm $i$ to an extent that can be quantified by $B_{i \mu}$, because it represents the dependency of firm $i$ on bank $\mu$ for the source of financing. Suppose additionally that a change in the level of firm $i$ 's financial degradation is quantified by another score, $y_{i}$, it would be reasonable to assume that $y_{i}$ is proportional to $B_{i \mu} x_{\mu}$ summed over banks $\mu$, or $y_{i} \propto \sum_{\mu} B_{i \mu} x_{\mu}$, as the influence from banks to firms. Fig. 5 (a) illustrates this direction of influence.

Similarly for the reverse direction of influence, from firms to banks. Firm $i$ with $y_{i}$ may delay its repayment, have defaults, even fail into bankruptcy, and so forth, due to its financial difficulties. Then the lending banks will not be able to fully enjoy profits in expected amounts due to the delay, may possibly have bad loans partially, if not totally, for the credit given to bankrupted firms. Any of them would result in the banks' financial deterioration, the level of which was assumed to be quantified by $x_{\mu}$ at the outset of our argument. Such influence to bank $\mu$ from firms is reasonably supposed to take the form, $x_{\mu} \propto \sum_{i} A_{\mu i} y_{i}$, in a similar way (see Fig. 5 (b) for illustration).

(a)

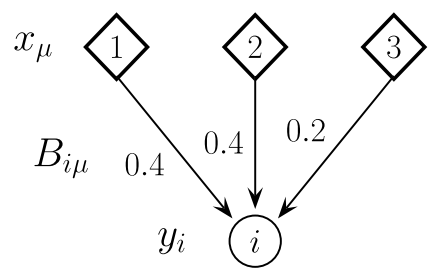

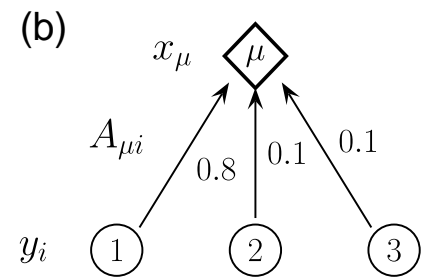

Figure 5: Illustration of (a) influence to firm $i$ from banks with an example of weights, $B_{i \mu}(\mu=1,2,3)$ satisfying Eq.(6), and of (b) influence to bank $\mu$ from firms with an example of weights, $A_{\mu i}(i=1,2,3)$ satisfying Eq.(4).

Expressing the change in the level of financial degradation of them as financial "fragility", our consideration above leads us to think about the influence from one score of fragility to the other by a set of equations which express the influence:

$$
\begin{aligned}
& \boldsymbol{y} \propto \mathbf{B} \boldsymbol{x}, \\
& \boldsymbol{x} \propto \mathbf{A} \boldsymbol{y},
\end{aligned}
$$

where $\boldsymbol{x}$ and $\boldsymbol{y}$ are the vectors with components, $x_{\mu}$ and $y_{i}$, respectively. It then follows that

$$
\mathbf{P} \boldsymbol{x}=\lambda \boldsymbol{x},
$$


where $\mathbf{P}:=\mathbf{A B}, \lambda$ is its eigenvalue and $\boldsymbol{x}$ is the corresponding eigenvector. $\boldsymbol{x}$ is the fragility scores of banks, and $\boldsymbol{y}$ is for firms.

Mathematically, an alternative set of scores could be defined, which we call "dual" scores. Namely, they are $u_{\mu}$ for bank $\mu$ and $v_{i}$ or firm $i$ which satisfy

$$
\begin{aligned}
\boldsymbol{v} & \propto \mathbf{A}^{\top} \boldsymbol{u}, \\
\boldsymbol{u} & \propto \mathbf{B}^{\top} \boldsymbol{v} .
\end{aligned}
$$

This leads to another eigenvalue problem, $\mathbf{P}^{\top} \boldsymbol{u}=\lambda \boldsymbol{u}$, or equivalently

$$
\boldsymbol{u}^{\top} \mathbf{P}=\lambda \boldsymbol{u}^{\top} .
$$

Here and hereafter, $T$ represents the transpose of a matrix or a vector, and we suppose a vector as a column vector by convention.

Thus, the set $\boldsymbol{x}$ of fragility scores of banks is the right eigenvector of the weight matrix $\mathbf{P}$ as in Eq.(9), and the set $\boldsymbol{u}$ of dual scores of banks satisfies the left eigenvector of $\mathbf{P}$ as in Eq.(12). Since the matrix $\mathbf{P}$ is not symmetric, one has a non-trivial relationship between $\boldsymbol{x}$ and $\boldsymbol{u}$ due to the definitions of $\mathbf{P}$ and the weight matrices of $\mathbf{A}$ and $\mathbf{B}$. In Appendix A:, we prove that the left eigenvalues and the right eigenvalues have a same spectrum, and that the left eigenvector $\boldsymbol{u}$ can be calculated from the right eigenvector $\boldsymbol{x}$ as in Eq.(A.5). We shall focus only on the fragility score in what follows.

As proved in Appendix A:, the eigenvalues and corresponding eigenvectors have the following mathematical properties.

- Spectrum of $\lambda$

$$
0<\lambda \leq 1 \text {. }
$$

- Trivial largest eigenvalue:

$$
\lambda=1 \text { if and only if } x_{\mu}=\text { constant. }
$$

- Summation formula of the eigenvalues:

$$
\sum_{k} \lambda_{k}=\sum_{\mu, i} A_{\mu i} B_{i \mu}=\operatorname{tr} \mathbf{P} .
$$

We can also interpret the definition of fragility scores in terms of dynamical propagation of influence. Let us consider a perturbation, or an idiosyncratic shock, that occurs with a configuration $\boldsymbol{x}$ among banks. It is assumed that the shock propagates by Eq.(7) to generate $\boldsymbol{y}$ among firms, which in turn affects the banks by Eq.(8). Although we do not have knowledge on the time-scale for this diffusion process, it would be reasonable to assume that the structure of credit network does not change much in the meanwhile. Then the propagation of the perturbation, going back and forth from banks to themselves, could be described by the repetition of Eq.(7) and Eq.(8), or equivalently, $\mathbf{P}^{r}$ for a finite number of iterations $r$.

Suppose that the eigenvalues are sorted in the decreasing order:

$$
1=\lambda_{1} \geq \lambda_{2} \geq \lambda_{3} \cdots \lambda_{n}>0 \text {. }
$$


The subspace spanned by the trivial eigenvector $\boldsymbol{x}^{(1)}$ should be ignored in the consideration of perturbation, since it merely represents a constant mode. Denote the resulting vector by $\tilde{\boldsymbol{x}}$, and expand it with respect to the non-trivial eigenvectors as $\tilde{\boldsymbol{x}}=\sum_{k=2}^{n} a_{k} \boldsymbol{x}^{(k)}$, then

$$
\begin{aligned}
\mathbf{P}^{r} \tilde{\boldsymbol{x}} & =\lambda_{2}^{r} a_{2} \boldsymbol{x}^{(2)}+\lambda_{3}^{r} a_{3} \boldsymbol{x}^{(3)}+\cdots+\lambda_{n}^{r} a_{n} \boldsymbol{x}^{(n)} \\
& =\lambda_{2}^{r}\left[a_{2} \boldsymbol{x}^{(2)}+\left(\frac{\lambda_{3}}{\lambda_{2}}\right)^{r} a_{3} \boldsymbol{x}^{(3)}+\cdots+\left(\frac{\lambda_{n}}{\lambda_{2}}\right)^{r} a_{n} \boldsymbol{x}^{(n)}\right] .
\end{aligned}
$$

This shows that the behavior of perturbation, in a long run $r \rightarrow \infty$, is determined mainly by the second largest eigenvalue and its corresponding eigenvector. For a finite $r$, it is suggested that one should consider only a few largest eigenvalues and the corresponding eigenvectors.

Therefore, the eigen decomposition of the idiosyncratic shocks, the profile of which is not known beforehand, can tell us which eigen-modes are important in the propagation of influence from banks to firms and vice versa in a finite time-scale.

\section{Results for the Dataset}

One needs to evaluate which eigen-modes are significant. In order to determine the significance of $\lambda_{2}, \lambda_{3}, \ldots$ and $\boldsymbol{x}^{(2)}, \boldsymbol{x}^{(3)}, \ldots$, we generate random bipartite graphs for comparison with the real data in the following way.

1. Cut every edge connecting bank $\mu$ and firm $i$. Then, for each original edge, we have two stubs; one from the bank (bank-stub) and the other from the firm (firm-stub).

2. Retain the original weight $w_{\mu i}$ on the $k_{\mu}$ stubs emanating from the bank $\mu$.

3. Randomly choose a pair of a bank-stub and a firm-stub, and rewire the pair by an edge.

The 3rd procedure is done so that there is no multiple edge between any pair of bank and firm. This rewiring procedure alters the weight as $w_{\mu i} \rightarrow w_{\mu j}$ if the edge emanating from $\mu$ to $i$ is randomly connected to $j$. Note that $w_{\mu}, k_{\mu}$ and $k_{i}$ are invariant for each $\mu$ and $i$ under rewiring, while $w_{i}$ becomes randomized. Therefore, the matrix $\mathbf{A}$ has the same structure except a permutation of columns. This means that a same amount of credit is supplied by a bank to a different firm in the randomly generated graphs.

The sum of eigenvalues satisfies Eq.(15). To compare the spectrum $\lambda$ with that for random graphs, one has to do so after a normalization. Define a normalized eigenvalue by

$$
\tilde{\lambda}_{k}=\frac{\lambda_{k}}{\sum_{\ell=1}^{n} \lambda_{\ell}}
$$

Fig. 6 (a) depicts the spectrum obtained for the credit network in the year 2005. By comparing with the spectrum for random graphs, we can say that only a few eigenvalues are significant. In this case, they are $\tilde{\lambda}_{2}$ and $\tilde{\lambda}_{3}$ (except $\tilde{\lambda}_{1}=\left(\sum_{\ell} \lambda_{\ell}\right)^{-1}$ ), while $\tilde{\lambda}_{7}$ and subsequent ones are indistinguishable from the spectrum for random graphs.

The corresponding eigenvectors $\boldsymbol{x}^{(2)}, \boldsymbol{x}^{(3)}, \ldots$ have components at a set of banks. To show this, the components $\left|x_{\mu}^{(2)}\right|$ is depicted in Fig. 6 (b). There are a few peaks at particular banks, while the same plot for random graphs (absolute value of each component averaged over 10 randomly generated graphs) is completely different from it. 
(a)

(b)
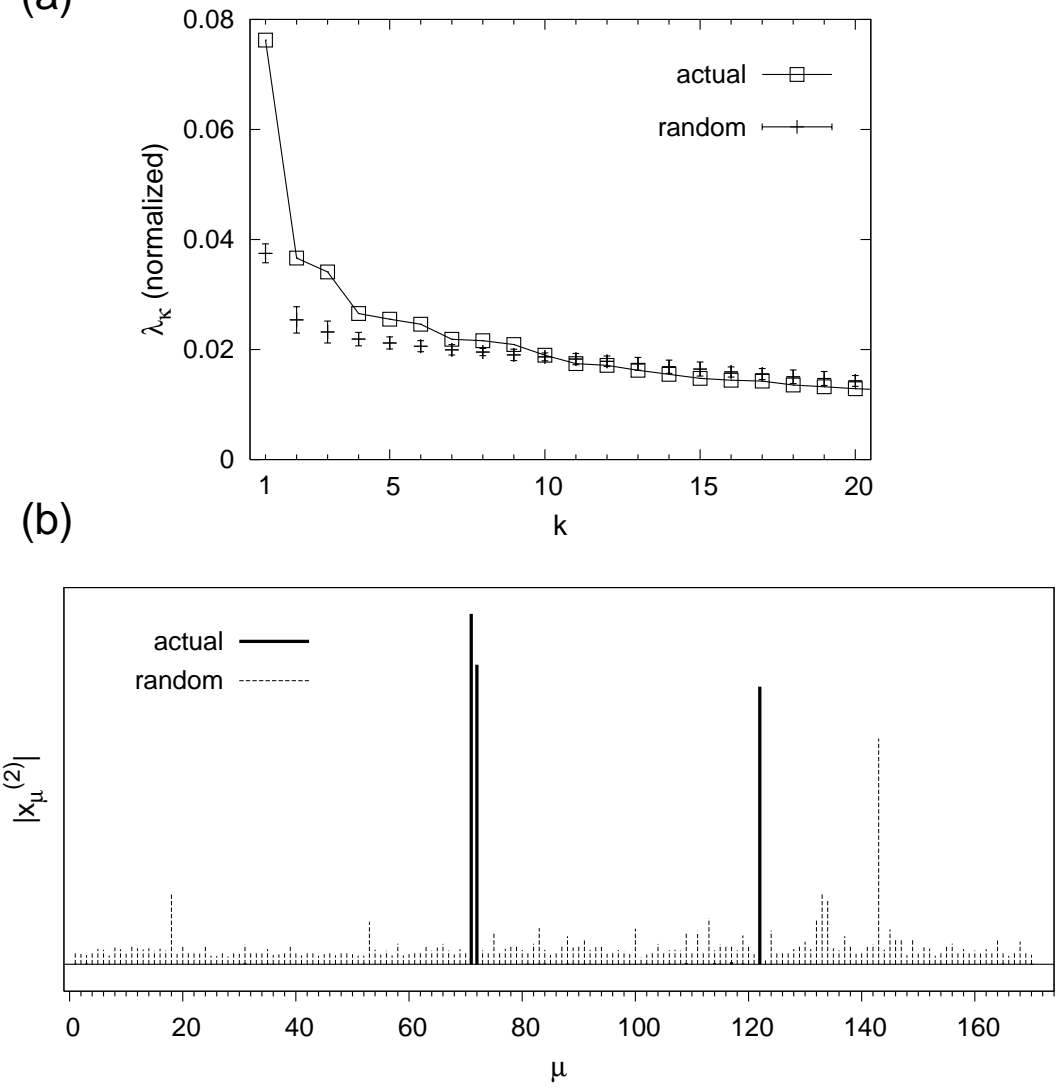

Figure 6: (a) Largest 20 eigenvalues $\tilde{\lambda}_{k}$ defined by Eq.(18). Squares are for the credit network in the year 2005. The points are averages each for 10 realizations of random bipartite graphs with the standard deviation. (b) The components of eigenvector $\left|x_{\mu}^{(2)}\right|$ for the actual data in the year 2005 (solid lines). Dotted lines show absolute values of components averaged over the random graphs.

This also demonstrates that these peaks of $\left|x_{\mu}^{(2)}\right|$ do not simply reflect the distribution for $w_{\mu}$, because under the randomization of bipartite graphs the configuration $w_{\mu}$ is not altered at all.

We also remark that if one simply takes into account of connectivity throwing away the information of weights, the resulting eigenvectors have quite different characteristics. This can be readily verified by assuming that $w_{\mu}=k_{\mu}$ and $w_{i}=k_{i}$, that is, by supposing that $w_{\mu i}=1$ for each edge.

For the historical data from 1980 to 2005, we obtained the spectrum in each year to see how the eigenvalues change in time. The result is shown in Fig. 7 for the largest two eigenvalues $\lambda_{2}$ and $\lambda_{3}$ normalized by Eq.(18). There are a strong peak in the late 80s and a drop in 1990; also two peaks around 1992 and in 1997.

The relationship between banks and firms changed in the course of the Japanese bubble (speculative investment into stocks and real estate) in the nation, notably in the late 80s 


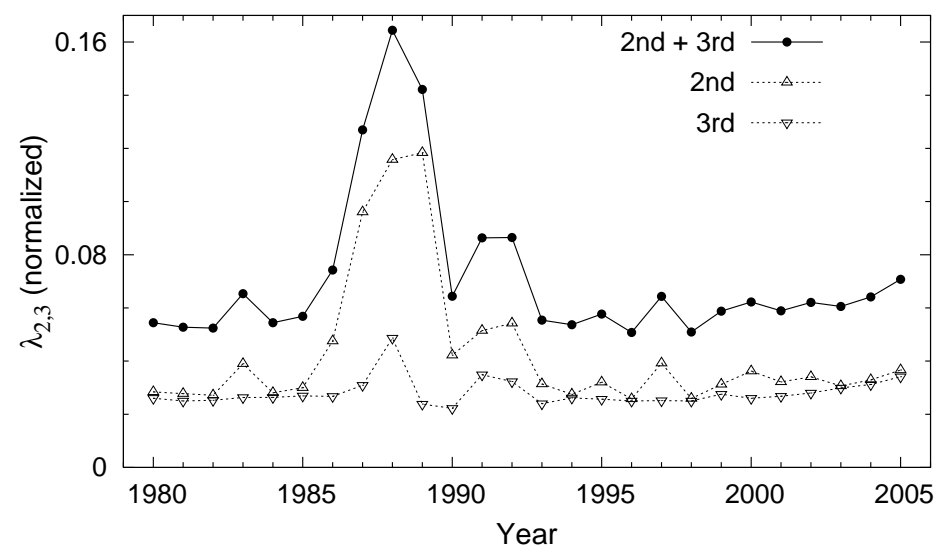

Figure 7: The sum (solid line) of the normalized eigenvalues $\tilde{\lambda}_{2}$ and $\tilde{\lambda}_{3}$, with their values (triangles and dotted lines) in each year from 1980 to 2005.

up to 1990 and after the bubble (after 1990) period. Two points should be considered for understanding what happened in the Japanese credit market during the period. First, firms were allowed to issue public debt, after financial deregulation, meaning that they were less dependent on bank loans. Secondly, after the collapse of bubble, banks were left with nonperforming loans, which hindered the intermediary role of banks. The problem of bad loans affected individual firm's decision to contract banks. It is known, for example, that during the bubble period the firms, especially large ones, tended to rely on a single relation, while in the period of long stagnation after the collapse of the bubble the average percentage of multiple contracts increases (Ogawa et al., 2007). Banks then spent a decade or longer in 90s to recover from bad loans experiencing a financial crisis for a couple of years from 1997. In the late of 1997 and in 1998, three major and other small banks failed. While two major banks were nationalized, and other political decisions were made for maintaining the stability of financial system, most banks, major and minor, decreased the supply of credit immediately; even by reducing existing loans to firms, most notably for small and medium-sized firms.

Our observation in Fig. 7, for the late 80s and an abrupt change in 1990, coincides with this historical change of the bank-firm relationship. The fragility score in terms of the nontrivial eigenvalues increased during the period when firms tended to have single relation. Also, in 1997, banks decreased the total amount of loan during the 90s in attempt to reduce bad loans systematically. This can be considered to decrease the diversity in the credit system, resulting in the increase of fragility score.

We also examined the components of eigenvectors, $\boldsymbol{x}^{(2)}$ and $\boldsymbol{x}^{(3)}$, in order to have a look at how stable or unstable the eigen-structure is during the same period of time. We take the average to have the information on how large the non-trivial eigenvalues are, in comparison with that for random graphs, which can measure the fragility of the credit network. Fig. 8 shows the average of $\left|x_{\mu}^{(2)}\right|$ and $\left|x_{\mu}^{(3)}\right|$ for all the existed banks $\mu$ (horizontally) in the years from 1980 to 2005 (vertically from top to bottom). We can observe stable and unstable periods, and also peaks at particular banks. Notably, unstable pattern can be observed in the late 80s coinciding the course of the bubble, and also in 90s after the bubble. There are peaks during these periods as well as for 1997 and 1998, which overlap the financial crisis. We shall discuss more about the results in the next section. 


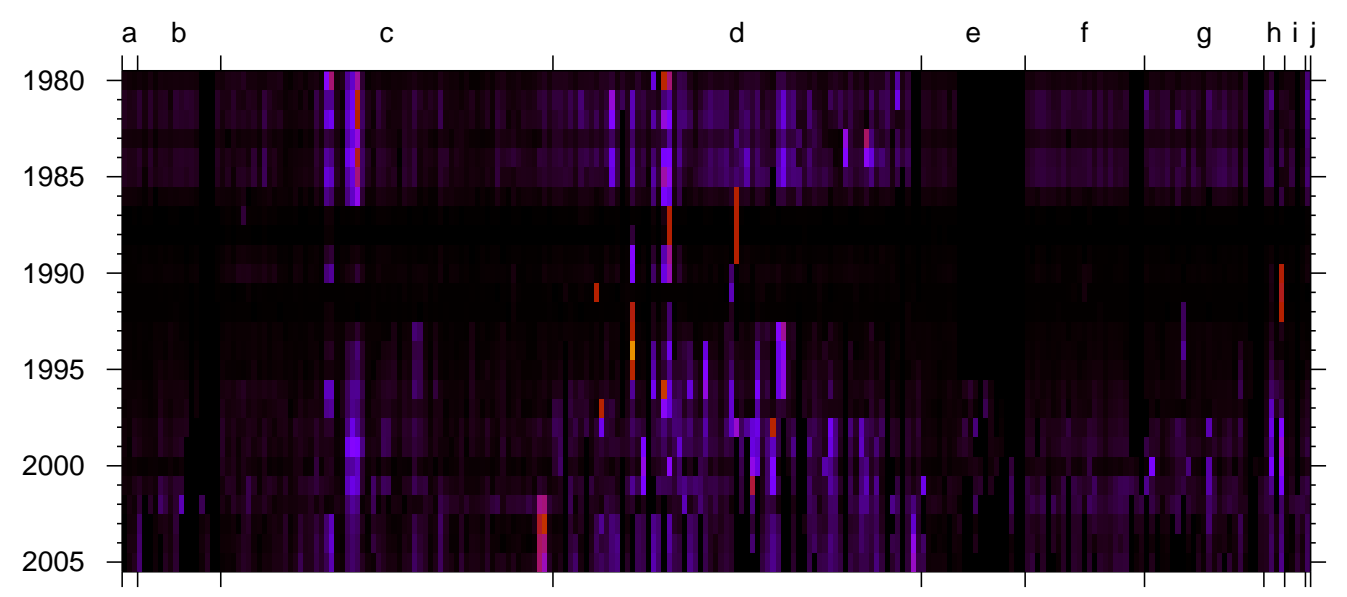

Figure 8: Components of the non-trivial eigenvectors, $\boldsymbol{x}^{(2)}$ and $\boldsymbol{x}^{(3)}$, corresponding to the largest two eigenvalues, during the years from 1980 (top) to 2005 (bottom). Each row represents the average of $\left|x_{\mu}^{(2)}\right|$ and $\left|x_{\mu}^{(3)}\right|$ by color, while columns are for $\mu=1, \ldots, n(n=229)$. See Table 1 for the classification of financial institutions, a (left) to $j$ (right). A cell's brighter color depicts a larger value.

\section{Discussion}

Fig. 7 and Fig. 8 describe the temporal change of the Japanese credit network with respect to the eigenvalues and corresponding eigenvectors. In order to fully understand our proposed scores of fragility for banks, one needs to compare the scores with the characteristics of financial conditions of banks, which can directly measure the level of financial deterioration. We shall investigate this point elsewhere. Here we relate the obtained results with historical description on the Japanese banking system in the past 25 years.

The absolute values of the eigenvectors, in Fig. 8, have a relatively stable profile among banks from 1980 to 1986 and from 2000 to 2005. The profile has peaks at several banks, notably a few regional banks (in the middle-north geographical region). In the late $80 \mathrm{~s}$, the profile changes dramatically, and spikes are present at two banks, from 1986 to 1989, which are in the middle-north region and are known to have deteriorated financially during the period. In the late $80 \mathrm{~s}$ to $90 \mathrm{~s}$, the Bank of Japan (BOJ) altered monetary policy tightening the policy most notably in 1990. After the bubble collapse, during the 90s, the profile changed into another configuration. A spike in the classification of $h$ refers to the Credit associations (Shinkin banks). Then, in the latter half of 90s, the profile went back to the previous one but with more peaks at other regional banks (especially at secondary regional banks). The spikes from 2003 to 2005 correspond to three banks in Okinawa.

Though we need more investigation beyond the anecdotal evidence, it is intriguing to note that several of the spikes in the profiles correspond to failed banks and to banks that had been merged into larger banks.

Also we note that the peaks and spikes mentioned above are present in same geographical regions - middle and north regions, and Okinawa. One of the authors (Y. F.) with collaborators recently showed that banks can be clustered into groups according to their patterns of lending to firms (De Masi et al., 2008). In fact, by defining the pattern for bank $\mu$ by a 
vector $\boldsymbol{a}_{\mu}$ that is equal to a column vector of the matrix $\mathbf{A}$ :

$$
\left(\boldsymbol{a}_{\mu}\right)_{i}:=A_{\mu i},
$$

it is possible to define a similarity in the lending patterns for a pair of banks $\mu$ and $\nu$, for example, by the inner product of the corresponding vectors $\boldsymbol{a}_{\mu}$ and $\boldsymbol{a}_{\nu}$. Then one can perform the clustering by standard methods including multi-dimensional scaling and hierarchical clustering. Indeed, De Masi et al. (2008) showed the minimum spanning tree (MST) calculated by a similarity measure ignoring the information of weight but considering only the connectivity from banks to firms. The resulting MST corresponds to clusters of cofinancing relationships of banks, which strongly reflect the geographical regions especially for the regional banks. It would be interesting to investigate how the eigen-structure is related to those clusters.

It is also remarked that, as described in Section II, we did not include the firms that went into bankruptcy. It should be interesting to include them in the credit network in order to evaluate the effect to banks and to compare the evaluation with the structural change that followed after the bankruptcy. It would be possible to model such propagation based on our consideration in defining the scores.

\section{Conclusion}

We studied the structure and its temporal change of Japanese credit relationships between commercial banks and quoted firms for the 25 years from 1980 to 2005. Each snapshot of the credit network is regarded as a weighted bipartite graph, where each node is either a bank or a firm, and an edge between a bank and a firm is defined to be present if there is a credit relationship between them. The edge has a weight that represents the amount of credit.

Suppose that a bank shrinks the amount of its supplied credit, a firm as debtor would be influenced to a certain extent that might be quantified by a matrix that can be calculated by the weight. Similarly, if a firm fails, then its effect to a bank as debtor would propagate to an extent that is measurable from the weight. To quantify the propagation, we introduced a set of score named fragility and its dual, and proved mathematical properties among them. The set of scores can be obtained by solving an eigenvalue problem.

By comparing the eigen-structure with that obtained in random bipartite graphs, which have same distributions for degrees of banks and firms and for normalized weight of banks, we found that the largest few (non-trivial) eigenvalues for the scores are significant. We performed historical analysis for our datasets, and showed that there are periods when the eigen-structure is stable or unstable, and that a particular set of banks, mostly a few regional banks, have large values of the fragility scores. Drastic change occurs in the late 80s during the bubble and also at the epochs of financially unstable periods including the financial crisis. Further investigation might be necessary to relate our results based on complex network analysis to the characteristic of banks, but we believe that our approach is a potentially valuable quantification of the structure and its temporal change of credit relationships. 


\section{Appendix A:. Mathematical properties of the eigenvalue problem}

As shown in Section B, the set $\boldsymbol{x}$ of fragility scores of banks is the right eigenvector of the weight matrix $\mathbf{P}$ as in Eq.(9), and the set $\boldsymbol{u}$ of dual scores of banks satisfy the left eigenvector of $\mathbf{P}$ as in Eq.(12). In this Appendix, we prove mathematical properties on eigenvalues and eigenvectors.

Let us first show that the score $\boldsymbol{u}$ can be calculated directly from the score $\boldsymbol{x}$. Eq.(9) is written explicitly in components as

$$
\frac{1}{w_{\mu}} \sum_{i, \nu} \frac{1}{w_{i}} w_{\mu i} w_{\nu i} x_{\nu}=\lambda x_{\mu},
$$

which we rewrite as

$$
\sum_{i, \nu} \frac{1}{w_{i}} w_{\mu i} w_{\nu i} x_{\nu}=\lambda w_{\mu} x_{\mu} .
$$

On the other hand, Eq.(12) is

$$
\sum_{\mu} u_{\mu} \frac{1}{w_{\mu}} \sum_{i} \frac{1}{w_{i}} w_{\mu i} w_{\nu i}=\lambda u_{\nu}
$$

which, after exchanging $\mu \leftrightarrow \nu$, reads as

$$
\sum_{i, \nu} \frac{1}{w_{i}} w_{\mu i} w_{\nu i} \frac{u_{\nu}}{w_{\nu}}=\lambda u_{\mu} .
$$

By comparing Eq.(A.2) and Eq.(A.4), we find that they are equivalent under the identification:

$$
u_{\mu} \propto w_{\mu} x_{\mu} .
$$

This also proves that left-eigenvalues and the right-eigenvalues have a same spectrum.

Let us consider two sets of eigenvalues and corresponding eigenvectors, $\left(\lambda^{(k)}, \boldsymbol{u}^{(k)}, \boldsymbol{x}^{(k)}\right)$ and $\left(\lambda^{(\ell)}, \boldsymbol{u}^{(\ell)}, \boldsymbol{x}^{(\ell)}\right)$. We have

$$
\boldsymbol{u}^{(k) \boldsymbol{\top}} \mathbf{P} \boldsymbol{x}^{(\ell)}=\lambda^{(k)} \boldsymbol{u}^{(k) \boldsymbol{\top}} \cdot \boldsymbol{x}^{(\ell)}=\lambda^{(\ell)} \boldsymbol{u}^{(k) \boldsymbol{\top}} \cdot \boldsymbol{x}^{(\ell)} .
$$

This means that

$$
0=\left(\lambda^{(k)}-\lambda^{(\ell)}\right) \boldsymbol{u}^{(k) \top} \cdot \boldsymbol{x}^{(\ell)}=\left(\lambda^{(k)}-\lambda^{(\ell)}\right) \sum_{\mu} u_{\mu}^{(k)} x_{\mu}^{(\ell)},
$$

which, by the use of Eq.(A.5), implies that

$$
0=\left(\lambda^{(k)}-\lambda^{(\ell)}\right) \sum_{\mu} w_{\mu} x_{\mu}^{(k)} x_{\mu}^{(\ell)} .
$$

Therefore, the eigenvectors should be orthonormal under the weight $w_{\mu}$ as a metric ${ }^{5}$. That is,

$$
\sum_{\mu} w_{\mu} x_{\mu}^{(k)} x_{\mu}^{(\ell)}=\delta_{k \ell}
$$

\footnotetext{
${ }^{5}$ Mathematically, $\boldsymbol{x}$ is a covariant vector, $\boldsymbol{u}$ is a contravariant vector, and the metric that connects them is given by $g_{\mu \nu}=\delta_{\mu \nu} w_{\mu}$. The orthogonalization of eigenvectors is done with respect to this metric.
} 
It follows from Eq.(A.9) the orthonormality:

$$
\sum_{k} w_{\mu} x_{\mu}^{(k)} x_{\nu}^{(k)}=\delta_{\mu \nu}
$$

This consideration of the inner product implies that we should take a look at the product of Eq.(A.2) and $x_{\mu}$. This leads us to

$$
\lambda=\frac{\sum_{i} \frac{1}{w_{i}}\left(\sum_{\mu} w_{\mu i} x_{\mu}\right)^{2}}{\sum_{\mu} w_{\mu} x_{\mu}^{2}} .
$$

This proves that $\lambda$ is real and positive, although the matrix $\mathbf{P}$ is not symmetric. Also we have the following inequality that holds for any value of $q$.

$$
0 \leq \sum_{\mu} w_{\mu i}\left(q-x_{\mu}\right)^{2}=w_{i} q^{2}-2\left(\sum_{\mu} w_{\mu i} x_{\mu}\right) q+\sum_{\mu} w_{\mu i} x_{\mu}^{2}
$$

This leads to the inequality for the discriminant:

$$
\left(\sum_{\mu} w_{\mu i} x_{\mu}\right)^{2}-w_{i} \sum_{\mu} w_{\mu i} x_{\mu}^{2} \leq 0
$$

from which it proves that the largest eigenvalue is 1.

$$
0<\lambda \leq 1 .
$$

This proves Eq.(13). It is obvious from Eq.(A.11) that $\lambda=1$ if and only if $x_{\mu}=q$. In fact, one can easily see, from Eq.(4) and Eq.(6) that $x_{\mu}=1(\mu=1, \ldots, n)$ is the eigenvector corresponding to $\lambda=1$, provided that the bipartite graph is connected (i.e. any node of bank or firm is reachable from any other $)^{6}$. This proves Eq.(14).

In addition, by applying the orthogonal relation in Eq.(A.10) to Eq.(A.2), it can be shown after a short calculation that the summation formula holds:

$$
\sum_{k} \lambda_{k}=\sum_{\mu, i} A_{\mu i} B_{i \mu}=\operatorname{tr} \mathbf{P} .
$$

This proves Eq.(15).

To summarize, the eigenvector $\boldsymbol{u}$ can be calculated directly from the eigenvector $\boldsymbol{x}$. Also the eigenvalues satisfy $0<\lambda \leq 1$, where the largest eigenvalue corresponds to a trivial eigenvector.

On the other hand, the dual scores, $\boldsymbol{u}$, corresponding to the largest eigenvalue $\lambda=1$ simply represents the total amount of loans, namely $u_{\mu} \propto w_{\mu}$ due to Eq.(A.5), so we can focus on non-trivial eigenvectors, $\boldsymbol{x}^{(2)}, \boldsymbol{x}^{(3)}$ and so on in the main text.

\footnotetext{
${ }^{6}$ For a disconnected graph, $x_{\mu}$ is constant in each connected components. The multiplicity of $\lambda=1$ is equal to the number of the connected components.
} 


\section{References}

Aoki, M. and H. Yoshikawa, Reconstructing Macroeconomics - A perspective from statistical physics and combinatorial stochastic processes, Cambridge University Press, 2007.

Brewer, E., H. Genay, and G. G. Kaufman, "Banking Relationships during Financial Distress: The Evidence from Japan," Economic Perspectives, 2003, 27 (3), 2-19.

Caldarelli, G., Scale-Free Networks, Oxford University Press, 2007.

De Masi, G. and M. Gallegati, "Bank-firm topology in Italy," 2007. submitted.

- , Y. Fujiwara, M. Gallegati, B. Greenwald, and J. E. Stiglitz, "An analysis of the Japanese credit network," 2008. arXiv:0901.2384v1 [q-fin.ST].

Delli Gatti, D., E. Gaffeo, M. Gallegati, G. Giulioni, and A. Palestrini, Emergent Macroeconomics: An Agent-based Apporoach to Business Fluctuations, Springer, 2008.

Imakubo, K. and Y. Soejima, "Network of fund transaction in call money market," Monetary and Economic Studies, Bank of Japan, 2008, 26. to appear.

Inaoka, H., H. Takayasu, T. Shimizu, T. Ninomiya, and K. Taniguchi, "Selfsimilarity of Banking Network," Physica A, 2004, 339, 621-634.

Iori, G., G. De Masi, O. Precup, G. Gabbi, and G. Caldarelli, "A Network Analysis of the Italian Overnight Money Market," 2007. to appear in Journal of Economic Dynamics and Control.

Kano, M., H. Uchida, G. F. Udell, and W. Watanabe, "Information Verifiability, Bank Organization, Bank Competition and Bank-Borrower Relationships," 2006. RIETI discussion paper 06-E-003, the Research Institute of Economy, Trade and Industry.

Ogawa, K., E. Sterken, and I. Tokutsu, "Why do Japanese firms prefer multiple bank relationship? Some evidence from firm-level data," Economic Systems, 2007, 31 (1), 49-70.

Uchida, H., G. F. Udell, and W. Watanabe, "Bank Size and Lending Relationships in Japan," Journal of the Japanese and International Economies, 2008, 22, 242-267. 